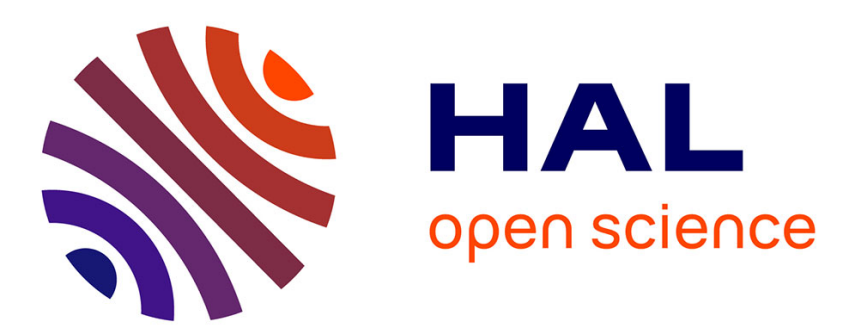

\title{
Comparaison de différentes méthodes de dosage des nitrates dans les extraits de sol. Influence du milieu d'extraction
}

Stephane Adamowicz, Christina Ottone, Simone Mars, Nicole Ballino

\section{To cite this version:}

Stephane Adamowicz, Christina Ottone, Simone Mars, Nicole Ballino. Comparaison de différentes méthodes de dosage des nitrates dans les extraits de sol. Influence du milieu d'extraction. Agronomie, 1982, 2 (4), pp.359-364. 10.1051/agro:19820408 . hal-01739307

\section{HAL Id: hal-01739307 \\ https://hal.science/hal-01739307}

Submitted on 20 Mar 2018

HAL is a multi-disciplinary open access archive for the deposit and dissemination of scientific research documents, whether they are published or not. The documents may come from teaching and research institutions in France or abroad, or from public or private research centers.
L'archive ouverte pluridisciplinaire HAL, est destinée au dépôt et à la diffusion de documents scientifiques de niveau recherche, publiés ou non, émanant des établissements d'enseignement et de recherche français ou étrangers, des laboratoires publics ou privés. 


\section{Comparaison de différentes méthodes de dosage des nitrates dans les extraits de sols. Influence du milieu d'extraction}

Stéphane ADAMOWICZ, Christiane OTTO, Simone MARS \& Nicole BALLINO

I.N.R.A., Station d'Agronomie, 45, boulevard du Cap, F 06602 Antibes.

RÉSUMÉ
Nitrates,
Sols,
Cadmium,
Hydrazine,
Electrode sélective
DEVARDA.

\begin{abstract}
Une comparaison de quatre méthodes de dosage des nitrates a été faite dans des extraits de quarante sols : la détermination directe à l'aide d'une électrode sélective, la méthode DEVARDA et deux méthodes automatiques basées sur la réduction des nitrates en nitrites, l'une par un mélange sulfate de cuivre-sulfate d'hydrazine, l'autre par une colonne de cadmium cuivré, et la colorimétrie des nitrites obtenus.

L'influence du milieu d'extraction $\left(\mathrm{KCl} 2 \mathrm{~N}, \mathrm{CaCl}_{2} 0,01 \mathrm{~N}\right.$ et $\left.\mathrm{CuSO}_{4} 0,02 \mathrm{~N}\right)$ sur les dosages de nitrates a été étudiée. Il a été vérifié qu'elle est nulle pour les dosages par la méthode DEVARDA et importante pour les autres méthodes.

Les méthodes colorimétriques automatiques ont donné des résultats satisfaisants dans l'extrait $\mathrm{KCl} 2 \mathrm{~N}$. Dans les extraits $\mathrm{CaCl}_{2} 0,01 \mathrm{~N}$ et $\mathrm{CuSO}_{4} 0,02 \mathrm{~N}$, nous avons constaté des interférences liées à la présence des ions calciques et cuivriques. Cependant, à l'exception de la méthode à l'hydrazine dans l'extrait $\mathrm{CuSO}_{4}$, les erreurs provoquées sont faibles pour les sols que nous avons étudiés et ces milieux semblent donc utilisables.

Pour les dosages potentiométriques, nous avons constaté des interférences importantes qui peuvent être éliminées totalement dans l'extrait $\mathrm{CaCl}_{2} 0,01 \mathrm{~N}$ par abaissement du $\mathrm{pH}$ à 4,5 par de l'acide sulfurique.

Cette précaution n'est pas nécessaire pour tous les modèles d'électrode de mesure entre lesquels d'importantes différences de sélectivité sont mises en évidence. Dans les extraits $\mathrm{CuSO}_{4} 0,02 \mathrm{~N}$, après abaissement $\mathrm{du} \mathrm{pH}$ à 4,5 , il subsiste une erreur dont nous n'expliquons pas l'origine.
\end{abstract}

\section{SUMMARY \\ Nitrates, \\ Soils, \\ Cadmium, \\ Hydrazine, \\ Ion sensitive electrode DEV ARDA.}

\section{Nitrate determination in soil extracts : Comparison of different methods and effect of the extraction medium}

Four nitrate determination methods were compared for 40 soil extracts : the DEVARDA method, potentiometry and two automatised colorimetric methods reducing nitrates to nitrites, either by a mixture of copper sulfate and hydrazine sulfate or by a copperized cadmium column.

Interactions with the extraction medium $\left(2 \mathrm{~N} \mathrm{KCl}, 0.01 \mathrm{~N} \mathrm{CaCl}_{2}\right.$ and $\left.0.02 \mathrm{~N} \mathrm{CuSO}_{4}\right)$ were studied. They did not influence results by the DEVARDA method but were of particular importance for potentiometry and automatic reduction to nitrites.

With autoanalysers, the best results, apparently free from interference, were obtained in the $\mathrm{KCl}$ extracts. Significant interference due to calcium and copper ions was established for the other two extraction media. However, except for the hydrazine method applied to $\mathrm{CuSO}_{4}$ extracts, the differences from the DEvaRDA values were small and these extracts appear to be suitable for nitrate determinations by automatic reduction to nitrite.

Potentiometric determinations were subject to severe interference, but this could be eliminated in $\mathrm{CaCl}_{2}$ extracts by lowering the $\mathrm{pH}$ to 4.5 with sulfuric acid. However this is not necessary for all electrode models, since these differ greatly in specificity. Lowering the $\mathrm{pH}$ to 4.5 in the $\mathrm{CuSO}_{4}$ extracts did not eliminate interference completely and significant differences from the DEVARDA method remained.

\section{INTRODUCTION}

Les dosages de nitrates dans les extraits de sol se font couramment par 3 types de méthodes :

- Dosage direct des nitrates par utilisation d'électrodes sélectives,

- Réduction des nitrates en ammonium d'après la méthode DEVARDA et titration après distillation de l'ammonium,

- Réduction des nitrates en nitrites et dosage colorimétrique de ces derniers selon la réaction de GRIESS.

Si la méthode DEvaRDA est généralement considérée comme une bonne technique de référence, elle présente cependant l'inconvénient d'être lente.

L'utilisation d'électrodes sélectives est séduisante par la 
simplicité du matériel nécessaire et la rapidité d'analyse qu'elle permet, mais peut être rendue délicate en raison de sa sensibilité à un grand nombre d'ions interférents. A ce propos, il est important de préciser le modèle et la marque d'électrode utilisés qui peuvent varier en spécificité (Goodman, 1976).

La plupart des mises au point de dosage des nitrates par potentiométrie ont été faites avec l'électrode ORION 92-07. Actuellement, ce fabricant commercialise une nouvelle sćrie 93-07 d'électrodes à module qui n'ont pas la même spécificité pour les nitrates et dont nous avons pu constater qu'elle donne des résultats sensiblement différents de l'ancien modèle 92-07. Il nous a donc paru important de définir les conditions d'utilisation de la nouvelle électrode 93-07.

Les méthodes colorimétriques utilisant le principe de la réduction des nitrates en nitrites posent des problèmes de reproductibilité lorsqu'on les réalise manuellement et ont tout intérêt à être automatisées. Elles autorisent alors de fortes cadences d'analyse sur des échantillons de faible volume (inférieur au $\mathrm{ml}$ ), tout en ne mobilisant que le minimum de main-d'œuvre. Ces qualités sont particulièrement appréciables dans les laboratoires traitant de grandes séries d'échantillons. Nous comparerons 2 types d'analyses différant par le réducteur employé. L'une utilise un mélange sulfate de cuivre-sulfate d'hydrazine (TERREY, 1966), l'autre une colonne de cadmium métallique avec dépôt de cuivre (Henriksen \& Selmer-Olsen, 1970).

Il nous a paru nécessaire de comparer les résultats obtenus par ces méthodes dans divers milieux d'extraction des nitrates $\left(\mathrm{KCl} 2 \mathrm{~N}, \mathrm{CaCl}_{2} 0,01 \mathrm{~N}, \mathrm{CuSO}_{4} 0,02 \mathrm{~N}\right)$. En effet, si l'extraction peut se faire par de l'eau (MILham et al., 1970), l'utilisation d'un milieu salin présente l'avantage de floculer les argiles, ce qui facilite la filtration et la clarification de l'extrait. Une solution de $\mathrm{KCl} 2 \mathrm{~N}$ (BREMNER, 1965) possède cette propriété et extrait l'ammonium échangeable en plus des nitrates. Cependant, sa teneur en chlorures ne permet pas les dosages par électrode sélective des nitrates ; c'est pourquoi nous utilisons depuis plusieurs années une extraction par $\mathrm{CaCl}_{2}$ 0,01 N (MAZOYER, 1972). $\mathrm{Ce}$ milieu flocule les argiles et permet les dosages potentiométriques avec une sensibilité suffisante. Afin de ralentir l'évolution de la teneur en nitrates de l'extrait, LEWIS (1961) a proposé une solution de $\mathrm{CuSO}_{4} 0,02 \mathrm{~N}$ et $\emptyset$ IEN \& SELMER-OLSEN (1969) ont montré que les dosages par électrode sélective sont possibles dans un tel milieu. Par contre, pour les méthodes utilisant le principe de la réduction des nitrates en nitrites, l'ion cuivrique a un rôle essentiel pour fixer le potentiel d'oxydo-réduction et nous cherchons à savoir dans quelle mesure l'utilisation de l'extrait $\mathrm{CuSO}_{4} 0,02 \mathrm{~N}$ peut interférer dans les dosages.

\section{MATÉRIELS ET MÉTHODES}

\section{A. Echantillonnage - Extraction}

Quarante échantillons de terres de texture et de composition variées (tabl. 1) ont été choisis parmi les collections de la station d'Antibes. Ils proviennent de sols de serre ou de plein air et ont été prélevés à diverses profondeurs dans les profils culturaux. Ils ont été séchés à température ambiante et tamisés à $2 \mathrm{~mm}$. L'extraction est faite par agitation pendant $1 \mathrm{~h}$ de $50 \mathrm{~g}$ de terre avec $250 \mathrm{ml}$ de $\mathrm{KCl} 2 \mathrm{~N}, 100 \mathrm{ml}$ de $\mathrm{CaCl}_{2} 0,01 \mathrm{~N}$ ou $100 \mathrm{ml}$ de $\mathrm{CuSO}_{4} 0,02 \mathrm{~N}$, selon les cas précisés dans le texte. Ces deux derniers rapports d'extraction sont calculés pour atteindre une sensibilité suffisante dans les dosages par électrode sélective. Les extraits sont ensuite filtrés et analysés simultanément par les différentes méthodes.

\section{B. Techniques analytiques}

Après dosage, un ajout de nitrates est fait dans chaque extrait et un nouveau dosage permet de calculer le pourcentage retrouvé des ajouts (A). Tout dosage (avec ou sans ajout) a été répété 3 fois, sauf par la méthode DEvarda en raison de sa lenteur.

Les gammes de nitrates servant à étalonner les appareils, ainsi que les ajouts de nitrates ont toujours été réalisés dans les mêmes milieux que les solutions d'extraction.

Tous les résultats sont exprimés en ppm d'azote nitrique dans la terre sèche. Le niveau de risque des tests statistiques (comparaisons de moyennes, régressions) a été fixé à 5 p. 100.

\section{Méthode DEVARDA}

Nous utilisons la méthode de BREMNER (1965) avec titration automatique. Un premier entraînement à la vapeur en présence de $500 \mathrm{mg}$ de magnésie calcinée permet d'éliminer l'ammonium de l'extrait ( 5 à $25 \mathrm{ml}$ ). Après refroidissement, un deuxième entraînement à la vapeur en présence d'alliage DEVARDA permet le dosage des nitrates réduits en ammonium. Celui-ci est recueilli dans $25 \mathrm{ml}$ d'acide borique à 1 p. 100 dans une solution éthanol/eau $(20 / 80 \mathrm{v} / \mathrm{v})$. Un dispositif automatique permet de titrer l'ammonium par $\mathrm{H}_{2} \mathrm{SO}_{4} 0,005 \mathrm{~N}$ au fur et à mesure de son déplacement, le $\mathrm{pH}$ étant ainsi maintenu à 5,2. Les quantités d'acide apportées sont enregistrées en fonction du temps ce qui permet d'apprécier le point final du dosage. La quantité d'alliage DEVARDA (MERCK) est de $200 \mathrm{mg}$ environ, mais lorsque le dégagement d'ammonium s'avère trop lent, cette quantité peut être augmentée.

\section{Potentiométrie}

Nous utilisons une électrode sélective ORION 93-07 à module et une électrode de référence TACUSSEL $\mathrm{S} 8$ (solution de remplissage : $\mathrm{K}_{2} \mathrm{SO}_{4}$ en solution saturée). Le $\mathrm{pH}$ mètre millivoltmètre utilisé est un TACUSSEL ISIS 4000 de 0,5 millivolt de précision. Les électrodes sont trempées dans $25 \mathrm{ml}$ d'extrait modérément agité et la lecture de la mesure est faite lorsque l'affichage digital a oscillé au moins 10 fois entre 2 valeurs consécutives.

Si nécessaire, les extraits de sols sont dilués dans des solutions de $\mathrm{CaCl}_{2} 0,01 \mathrm{~N}$ ou $\mathrm{CuSO}_{4} 0,02 \mathrm{~N}$, selon les cas, de façon à ce que la teneur finale soit inférieure à $50 \mathrm{mg}$ d'azote nitrique par litre. Dans ces conditions, la force ionique de l'extrait à doser reste peu différente de celle des étalons et de la solution d'extraction. Une courbe d'étalonnage est établie entre 1 et $50 \mathrm{mg} \cdot \mathrm{1}^{-1}$ d'azote nitrique dans les milieux $\mathrm{CaCl}_{2} 0,01 \mathrm{~N}$ et $\mathrm{CuSO}_{4} 0,02 \mathrm{~N}$.

\section{Méthodes automatiques}

Nous avons utilisé 2 autoanalyseurs à flux segmenté par de l'air qui réduisent les nitrates en nitrites et dosent ces derniers colorimétriquement selon la réaction de GRIESS (SNELL \& SNELL, 1949). L'un est une chaîne QUANTA avec dialyseur dont nous avons modifié le manifold (fig. 1). La réduction des nitrates en nitrites est faite par un mélange sulfate d'hydrazine-sulfate de cuivre (TERREY, 1966). Les réactifs sont décrits dans le tableau 2. Les échantillons sont dosés dans la gamme de 1 à $25 \mathrm{mg}$ d'azote nitrique par litre. 
TABLEAU 1

Composition des 40 terres analysées.

Mean characteristics of the 40 soils analysed.

\begin{tabular}{|c|c|c|c|c|c|c|c|}
\hline $\mathbf{N}^{0}$ & $\begin{array}{l}\mathrm{N} \text { total } \\
\text { p. mille }\end{array}$ & $\begin{array}{c}\text { Calcaire } \\
\text { total } \\
\text { p. mille }\end{array}$ & $\begin{array}{l}\text { Calcaire } \\
\text { actif } \\
\text { p. mille }\end{array}$ & pH eau & $\mathrm{pH} \mathrm{KCl}$ & $\begin{array}{c}\mathrm{Cl}^{-} \\
\text {p.p.m. }\end{array}$ & $\begin{array}{l}\text { C.E.C. } \\
\text { me p. } 100 \mathrm{~g}\end{array}$ \\
\hline 1 & 0,06 & 116 & 26 & 8,2 & 7,1 & - & 8,7 \\
\hline 2 & 0,28 & 0 & 0 & - & - & 0 & - \\
\hline 3 & 0,29 & 0 & 0 & 5,2 & 4,1 & 0 & - \\
\hline 4 & 0,45 & 4 & 0 & 7,8 & 7,1 & 78 & - \\
\hline 5 & 0,47 & 206 & 38 & 8,4 & 7,6 & 0 & 4,5 \\
\hline 6 & 0,52 & - & 9 & 7,7 & 7,0 & 130 & 10,5 \\
\hline 7 & 0,58 & 11 & 0 & 7,7 & 7,2 & - & 8,8 \\
\hline 8 & 0,58 & 482 & 157 & 8,1 & 7,4 & - & 7,6 \\
\hline 9 & 0,61 & 126 & 25 & 8,1 & 7,5 & 0 & - \\
\hline 10 & 0,61 & 17 & 6 & 8,2 & 7,4 & 0 & 9,7 \\
\hline 11 & 0,62 & 17 & 6 & 8,2 & 7,4 & - & 8,2 \\
\hline 12 & 0,62 & 17 & 6 & 8,2 & 7,4 & 186 & 9,0 \\
\hline 13 & 0,65 & 17 & 6 & 8,2 & 7,4 & 122 & 9,7 \\
\hline 14 & 0,66 & 0 & 0 & 8,2 & 7,2 & 0 & 12,4 \\
\hline 15 & 0,71 & 576 & 42 & 8,4 & 7,6 & 0 & - \\
\hline 16 & 0,76 & 16 & 12 & 7,4 & 6,6 & 64 & 14,6 \\
\hline 17 & 0,81 & 98 & 47 & 8,1 & 7,1 & - & 22,1 \\
\hline 18 & 0,86 & 44 & 5 & 8,0 & 7,5 & - & 7,9 \\
\hline 19 & 0,92 & 0 & 0 & 7,7 & 6,6 & - & 20,0 \\
\hline 20 & 1,10 & 34 & 7 & 8,1 & 7,3 & 0 & - \\
\hline 21 & 1,20 & 237 & 35 & 8,3 & 7,4 & 0 & - \\
\hline 22 & 1,23 & 4 & 0 & 7,0 & 6,7 & 452 & 14,2 \\
\hline 23 & 1,26 & - & 9 & 7,0 & 6,5 & - & 15,5 \\
\hline 24 & 1,31 & 118 & 25 & 7,9 & 7,5 & 568 & 9,0 \\
\hline 25 & 1,33 & 64 & 16 & 8,2 & 7,4 & - & 10,0 \\
\hline 26 & 1,34 & 89 & 17 & 8,3 & 7,5 & 150 & 10,0 \\
\hline 27 & 1,40 & 136 & 40 & 7,9 & 7,5 & 158 & 10,0 \\
\hline 28 & 1,72 & 383 & 31 & 8,2 & 7,4 & 0 & - \\
\hline 29 & 1,89 & 0 & 0 & 7,8 & 7,4 & - & - \\
\hline 30 & 1,94 & 0 & 0 & 7,8 & 7,4 & 72 & - \\
\hline 31 & 2,16 & 38 & 18 & 7,7 & 7,1 & - & - \\
\hline 32 & 2,18 & 0 & 0 & 6,9 & 6,6 & 0 & - \\
\hline 33 & 2,18 & 37 & 17 & 7,8 & 7,1 & - & - \\
\hline 34 & 2,23 & 0 & 0 & 4,6 & 3,7 & 0 & 15 \\
\hline 35 & 2,42 & 43 & 20 & 7,8 & 7,2 & - & - \\
\hline 36 & 2,59 & 424 & 25 & 7,7 & 7,3 & 400 & - \\
\hline 37 & 3,02 & 400 & 132 & 7,4 & 6,8 & 238 & 16,8 \\
\hline 38 & 3,56 & 359 & 45 & 7,7 & 7,2 & 90 & - \\
\hline 39 & 4,50 & 144 & 55 & 7,9 & 7,3 & - & 34 \\
\hline 40 & 4,59 & 16 & 6 & 7,2 & 6,8 & - & 27,9 \\
\hline
\end{tabular}

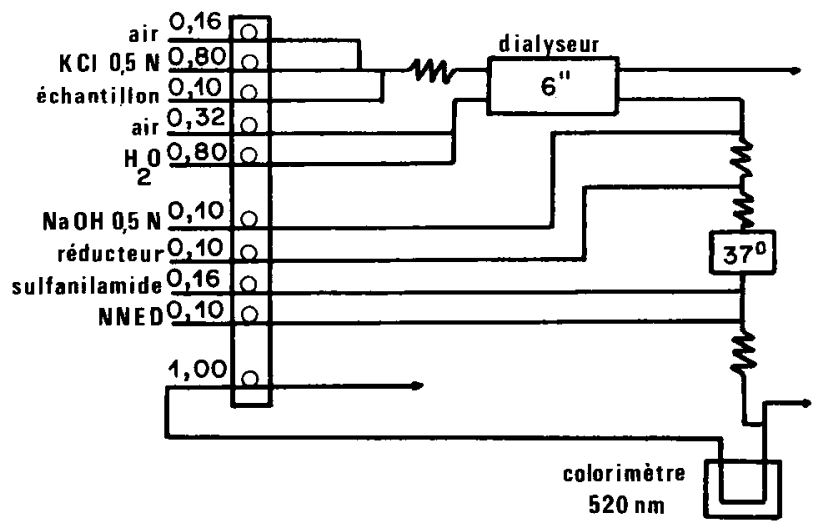

Figure 1

Schéma de l'analyseur de nitrates par la méthode à l'hydrazine, les débits sont en $m l . m n^{-1}$.

Manifold for nitrate determinations by the hydrazine method, flow rates in $m l . m n^{-1}$.

L'autre chaîne (fig. 2, tabl. 2) a été réalisée au laboratoire. Elle n'est pas équipée de dialyseur et utilise une

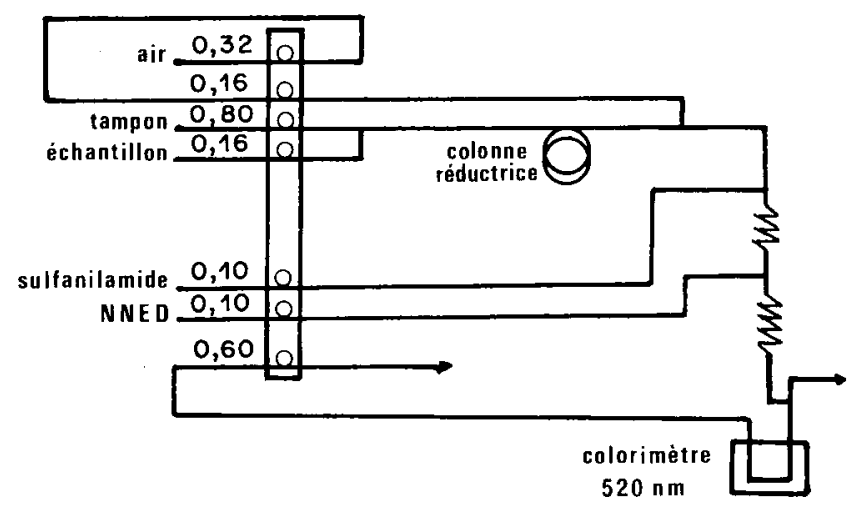

Figure 2

Schéma de l'analyseur de nitrates par la méthode au cadmium, les débits sont en $\mathrm{ml} . \mathrm{mn}^{-1}$.

Manifold for nitrate determinations by the cadmium method, flow rates in $m l . m n^{-1}$.

colonne de cadmium cuivré préparée selon STAINTON (1974) pour réduire les nitrates en nitrites (HENRIKSEN \& SELMEROLSEN, 1970). Le montage initialement réalisé pour doser 
TABLEAU 2

Composition des réactifs pour les analyseurs automatiques.

Reagents for autoanalysers.

\begin{tabular}{|c|c|c|}
\hline Réactifs & Méthode à l'hydrazine & Méthode au cadmium \\
\hline Tampon & & $\begin{array}{l}\mathrm{NH}_{4} \mathrm{Cl}: 20 \mathrm{~g} \\
\mathrm{Na} \text { tétraborate }: 4 \mathrm{~g} \\
\text { EDTA : } 0,2 \mathrm{~g} \\
\text { Acide oxalique : } 0,25 \mathrm{~g} \\
\text { pH élevé à } 9,6 \text { par } \mathrm{NH}_{4} \mathrm{OH} \\
\mathrm{H}_{2} \mathrm{O}: \text { q.s.p. } 11\end{array}$ \\
\hline Agent réducteur & $\begin{array}{l}\text { Sulfate d'hydrazine : } 180 \mathrm{mg} \\
\text { Sulfate de cuivre : } 1,6 \mathrm{mg} \mathrm{H} \mathrm{H}_{2} \mathrm{O}: 100 \mathrm{ml}\end{array}$ & $\begin{array}{l}1 \mathrm{~m} \text { de fil de cadmium } \\
\text { préparé selon Stainton (1974) }\end{array}$ \\
\hline Sulfanilamide & $\begin{array}{l}\text { Sulfanilamide }: 10 \mathrm{~g} \\
\mathrm{HCl}: 200 \mathrm{ml} \\
\mathrm{H}_{2} \mathrm{O}: 800 \mathrm{ml}\end{array}$ & idem \\
\hline NNED & $\mathrm{N}(1-\mathrm{Naphtyl})$ Ethylene di-amine dihydrochloride : $1 \mathrm{~g} \mathrm{H}_{2} \mathrm{O}: 11$ & idem \\
\hline
\end{tabular}

de très faibles concentrations en azote nitrique (20 à $500 \mu \mathrm{g} \cdot \mathrm{1}^{-1}$ ) nécessite une prédilution manuelle importante des extraits ( 200 fois généralement).

Il a été contrôlé qu'aucun échantillon ne contient de nitrites.

\section{RÉSULTATS ET DISCUSSION}

Les échantillons couvrent assez uniformément la gamme de 0 à $150 \mathrm{ppm}$, plus un de $500 \mathrm{ppm}$. La présence de cet échantillon n'a pas particulièrement modifié les résultats présentés.

1. Les moyennes des dosages des 40 terres par les différentes méthodes dans les 3 milieux d'extraction ont été classées dans le tableau 3 par ordre croissant. Il est vérifié

\section{TABLEAU 3}

Classement des moyennes de dosage d'azote nitrique dans 40 sols. Les valeurs suivies d'une même lettre ne sont pas significativement différentes à $P=0,05$. A est le pourcentage retrouvé des ajouts, - indique qu'il est significativement différent de 100 ou que $R$ représente une liaison significative entre l'erreur $E$ par rapport au DEVARDA et la teneur en calcaire actif.

Classification of the mean determinations of $\mathrm{NO}_{3}-\mathrm{N}$ in 40 soils. Values followed by the same letter are not significantly different at $P=0.05 . A$ is the percent of $\mathrm{NO}_{3}$ recovery, indicates that it is significantly different from $100 . R$ is the correlation coefficient between $E$ and active lime, ${ }^{\circ}$ indicates that the correlation is significant.

\begin{tabular}{|c|c|c|c|c|}
\hline Méthode & $\begin{array}{c}\text { Milieu } \\
\text { d'extraction }\end{array}$ & $\begin{array}{c}\text { Moyennes } \\
\text { ppm }\end{array}$ & A & $\mathrm{R}^{2} \times 100$ \\
\hline DEVARDA & $\mathrm{KCl}$ & $58,97 \mathrm{a}$ & 99,2 & - \\
\hline DEVARDA & $\mathrm{CuSO}_{4}$ & $59,08 \mathrm{a}$ & 100,1 & - \\
\hline Cadmium & $\mathrm{CuSO}_{4}$ & 59,93 a b & 99,1 & $25,6^{\circ}$ \\
\hline DEVARDA & $\mathrm{CaCl}_{2}$ & $59,95 \mathrm{a} \mathrm{b}$ & 96,0 & - \\
\hline Hydrazine & $\mathrm{KCl}^{2}$ & $60,67 \quad b$ & 100,5 & 0,4 \\
\hline Cadmium & $\mathrm{KCl}$ & $60,97 \mathrm{~b}$ & 98,5 & 0,5 \\
\hline Hydrazine & $\mathrm{CaCl}_{2}$ & $62,81 \mathrm{c} \mathrm{b}$ & $98,6^{\circ}$ & $11,1^{\circ}$ \\
\hline Cadmium & $\mathrm{CaCl}_{2}$ & $63,07 \mathrm{c}$ & 99,9 & $10,7^{\circ}$ \\
\hline Hydrazine & $\mathrm{CuSO}_{4}$ & $67,06 \mathrm{~d}$ & $112,3^{\circ}$ & $14,3^{\circ}$ \\
\hline Potentiométrie & $\mathrm{CaCl}_{2}$ & $68,15 \mathrm{~d}$ & $106,4^{\circ}$ & $12,8^{\circ}$ \\
\hline Potentiométrie & $\mathrm{CuSO}_{4}$ & $74,56 \mathrm{e}$ & $123,2^{\circ}$ & $11,7^{\circ}$ \\
\hline
\end{tabular}

que la méthode DEVARDA donne des résultats équivalents dans les divers extraits, ce qui est confirmé par les paramètres des droites de régression du tableau 4. Les pentes de ces droites ne sont pas significativement différentes de 1 et nous pouvons conclure que le rapport terre sur solution et la nature du milieu d'extraction n'ont pas influencé de façon mesurable les quantités de nitrates extraites (MiLhaM et al., 1970 ; Anonyme, 1978).

\section{TABLEAU 4}

Régression linéaire $Y=a X+b$ entre les dosages de nitrates par la méthode DEVARDA dans divers milieux d'extraction. $X$ représente les dosages dans l'extrait $\mathrm{KCl} 2 \mathrm{~N}$.

Linear regression analysis $Y=a X+b$ of nitrate determinations $b y$ the DEVARDA method in different media. $X$ is the result in $\mathrm{KCl}$ extract.

\begin{tabular}{cccc}
\hline $\mathrm{Y}$ & $\mathrm{a}$ & $\mathrm{b}$ & $\mathbf{R}^{2} \times 100$ \\
\hline Milieu $\mathrm{CaCl}_{2}$ & 1,015 & 0,071 & 99,81 \\
Milieu $\mathrm{CuSO}_{4}$ & 0,993 & 0,519 & 99,88 \\
\hline
\end{tabular}

Par ailleurs, le tableau 3 indique que le pourcentage retrouvé des ajouts n'est pas significativement différent de 100 et qu'il n'y a pas d'interférences mesurables par cette méthode.

Nous considérons donc par la suite que la méthode DEVARDA peut être prise comme référence et nous appellerons erreurs $(\mathrm{E})$ les écarts de dosage des autres méthodes par rapport à celle-ci.

2. D'après les paramètres des droites de régression des autres techniques par rapport au DEVARDA (tabl. 5), il apparaît que seules les colorimétries automatiques dans l'extrait $\mathrm{KCl}$ donnent des pentes non significativement différentes de 1 et des pourcentages retrouvés des ajouts non significativement différents de 100 (tabl. 3). Il ne semble donc pas y avoir d'interférences mesurables et nous admettons que ces méthodes donnent des valeurs par excès de 2 ppm environ par rapport au DEVARDA (tabl. 3), ce qui est négligeable par rapport à la moyenne des dosages.

3. Dans les milieux $\mathrm{CaCl}_{2}$ et $\mathrm{CuSO}_{4}$, la potentiométrie et les colorimétries automatiques conduisent à des droites de 
TABLEAU 5

Régression linéaire $Y=a X+b$ entre une méthode de dosage des nitrates $Y$ et le DEVARDA $(X) .{ }^{\circ}$ indique que la pente a est significativement différente de 1 à $P=0,05$.

Linear regression analysis $Y=a X+b . Y$ is the method compared to DEVARDA $(X) .{ }^{\circ}$ indicates that a is significantly different from 1 at $P=0.05$

\begin{tabular}{clrrr}
\hline \hline $\begin{array}{c}\text { Milieu } \\
\text { d'extraction }\end{array}$ & Méthode $\mathrm{Y}$ & a & b & $\mathrm{R}^{2} \times 100$ \\
\hline \multirow{2}{*}{$\mathrm{KCl}$} & Hydrazine & 1,000 & 1,67 & 99,89 \\
& Cadmium & 1,015 & 1,10 & 99,85 \\
& Cadmium & $1,061^{\circ}$ & $-0,61$ & 99,91 \\
$\mathrm{CaCl}_{2}$ & Hydrazine & $1,075^{\circ}$ & $-1,78$ & 99,92 \\
& Potentiométrie & $1,106^{\circ}$ & $\mathbf{1 , 8 3}$ & 99,96 \\
& Cadmium & $1,048^{\circ}$ & $-1,75$ & 99,54 \\
$\mathrm{CuSO}_{4}$ & Hydrazine & $1,084^{\circ}$ & 3,04 & 99,89 \\
& Potentiométrie & $1,108^{\circ}$ & 8,92 & 99,56 \\
& & & & \\
\hline
\end{tabular}

régression par rapport au DEVARDA de pentes significativement supérieures à 1. Par ailleurs, l'erreur E est toujours corrélée à la teneur en calcaire actif comme l'indique le tableau 3. Il y aurait donc des interférences, confirmées en ce qui concerne la potentiométrie et la méthode à l'hydrazine par des pourcentages retrouvés des ajouts significativement différents de 100 (tabl. 3).

- Pour les colorimétries automatiques, les interférences dans l'extrait $\mathrm{CaCl}_{2}$ peuvent s'expliquer par des précipitations d'hydroxydes de calcium au cours de l'étape de réduction des nitrates en nitrites qui se fait à un $\mathrm{pH}$ supérieur à 9, comme l'a déjà signalé TERREY (1966). Cependant, pour les échantillons de teneurs supérieures à 2 ppm, l'erreur relative n'a été en moyenne que de 3 p. 100 pour la méthode au cadmium et de $5 \mathrm{p} .100$ pour la méthode à l'hydrazine.

Par contre, dans l'extrait $\mathrm{CuSO}_{4}$, l'erreur relative a été de 20 p. 100 en moyenne pour la méthode à l'hydrazine. Cette erreur peut être attribuée aux ions cuivriques de l'extrait même. Ils interviennent dans une proportion précise dans le mélange réducteur (tabl. 2) et leurs teneurs sont variables dans l'extrait en raison d'adsorption par les argiles et les matières organiques et éventuellement de précipitation par les carbonates. Nous avons vérifié sur 16 de nos extraits que la teneur en ion cuivrique varie de 0,5 à $20 \mathrm{mg} \cdot 1^{-1}$, ce qui est faible en comparaison des $635 \mathrm{mg} . \mathrm{l}^{-1}$ initialement présents dans la solution d'extraction. Nous avons établi une corrélation significative entre l'erreur et la teneur résiduelle en $\mathrm{Cu}^{++}$de l'extrait ( $\left.\mathrm{R}=-0,57,14 \mathrm{DDL}\right)$. Pour confirmer l'influence des ions cuivriques de la solution, nous avons dosé les nitrates de 17 extraits de sol dans $\mathrm{CuSO}_{4}$ $0,02 \mathrm{~N}$ en étalonnant l'analyseur d'une part, sur une gamme de nitrates faite dans une solution de $\mathrm{CuSO}_{4}, 0,02 \mathrm{~N}$ (selon matériel et méthodes) et d'autre part, sur une gamme de nitrates dans l'eau. Les droites de régression par rapport au DEVARDA (X) sont les suivantes :

$$
\begin{aligned}
& \text { - gamme } \mathrm{CuSO}_{4} 0,02 \mathrm{~N} \\
& \mathrm{Y}=1,116 \mathrm{X}+2,37 \quad\left(\mathrm{R}^{2}=0,9971\right) \\
& \text { - gamme } \mathrm{H}_{2} \mathrm{O} \\
& \mathrm{Y}=1,033 \mathrm{X}-0,58 \quad\left(\mathrm{R}^{2}=0,9980\right) .
\end{aligned}
$$

Seule la pente de la première de ces droites est significativement supérieure à 1 . Il est donc vraisemblable que c'est la grande différence de teneur en ions cuivriques entre les extraits de sol et les solutions d'étalonnage qui est responsa- ble de l'erreur observée. Bien que plus faibles, les erreurs constatées dans l'extrait $\mathrm{CuSO}_{4}$ avec la méthode au cadmium ( 3 p. 100 ) ont probablement la même origine. Les ions cuivriques interviennent dans le potentiel d'oxydoréduction et de plus déplacent le cadmium de la colonne réductrice, en modifiant ainsi l'efficacité.

- La potentiométrie est de loin la méthode ayant donné les fésultats les plus éloignés du DEvaRda (tabl. 3 et 5 ). Les moyennes des erreurs relatives des échantillons de teneur supérieure à $5 \mathrm{ppm}$ sont de 20 et 50 p. 100 dans les extraits $\mathrm{CaCl}_{2}$ et $\mathrm{CuSO}_{4}$ respectivement. L'erreur par excès est positivement corrélée à la conductivité des extraits, à la teneur en calcaire actif et à la teneur en nitrates.

Il ne s'agit donc pas d'une erreur due aux variations du coefficient d'activité des nitrates, celui-ci diminuant lorsque la force ionique augmente. Par ailleurs, quelques essais ponctuels de dosage par l'électrode ORION 92-07 sur quelques-uns de nos extraits $\mathrm{CaCl}_{2}$ ont donné des résultats comparables au DEVARDA, ce qui confirme les résultats obtenus par MAZOYER (1972).

Les erreurs constatées sont donc probablement dues à un défaut de spécificité de l'électrode 93-07.

Selon le manuel d'instruction de l'électrode, les acides organiques sont susceptibles d'interférer. Nous avons pu effectivement vérifier sur des solutions d'acides fulviques préparés à partir de tourbe que ceux-ci donnent une interférence positive dans le dosage des nitrates. Il reste cependant difficile d'évaluer l'ordre de grandeur de l'interférence qu'ils peuvent provoquer dans nos extraits de sols. Par ailleurs, l'erreur dans les extraits $\mathrm{CaCl}_{2}$ et $\mathrm{CuSO}_{4}$ se trouvant positivement corrélée à la teneur en calcaire actif du sol, une interférence par les bicarbonates des extraits s'avère possible. Pour éprouver ces deux hypothèses d'interférences, nous avons abaissé à une valeur comprise entre 4 et 4,5 , le $\mathrm{pH}$ des extraits filtrés, par des additions de $\mathrm{H}_{2} \mathrm{SO}_{4}$ comme le conseille le manuel d'instruction de l'électrode. Cette opération permet d'éliminer les bicarbonates et de floculer une partie des acides fulviques. Les quantités de $\mathrm{H}_{2} \mathrm{SO}_{4}$ nécessaires ont été inférieures à 1 millimole par litre d'extrait de sol.

On voit en comparant les tableaux 5 et 6 que, dans les 2 types d'extrait, l'abaissement du $\mathrm{pH}$ améliore considérablement les dosages par potentiométrie. Dans l'extrait $\mathrm{CaCl}_{2}$, les résultats d'analyses sont comparables au DEVARDA et le pourcentage retrouvé des ajouts n'est pas significativement différent de 100. Par contre, dans l'extrait $\mathrm{CuSO}_{4}$, la pente de la droite est significativement supérieure à 1 et le pourcentage retrouvé des ajouts (112 p. 100) est significativement supérieure à 100 . Bien que positivement corrélée à la teneur en chlorures, nous n'expliquons pas l'origine de cette interférence résiduelle. En effet, les

\section{TABLEAU 6}

Régressions linéaires $Y=a X+b$ entre la potentiométrie $(Y)$ sur des extraits dont le pH a été abaissé à 4,5 et le DEVARDA $(X) .{ }^{\circ}$ indique que la pente a est significativement différente de 1 à $P=0,05$.

Linear regression analysis $Y=a X+b$ between potentiometric

\begin{tabular}{|c|c|c|c|c|}
\hline $\begin{array}{c}\text { Milieu } \\
\text { d'extraction }\end{array}$ & a & b & $\mathrm{R}^{2} \times 100$ & D.D.L. \\
\hline $\mathrm{CaCl}_{2}$ & 1,000 & $-0,77$ & 99,60 & 21 \\
\hline $\mathrm{CuSO}_{4}$ & $1,050^{\circ}$ & $+2,97$ & 99,80 & 30 \\
\hline
\end{tabular}
determinations $(Y)$ in extracts after lowering $p H$ to 4.5 and $D E V A R D A$ method $(X) .{ }^{\circ}$ indicates that $a$ is significantly different from 1 at $P=0.05$. 
chlorures auraient dû interférer de la même façon dans les extraits $\mathrm{CaCl}_{2}$ et $\mathrm{CuSO}_{4}$ s'ils étaient en teneur suffisante. De plus, la teneur en chlorures étant significativement corrélée à la teneur en nitrates $\left(\mathrm{R}^{2}=0,373,23 \mathrm{DDL}\right)$, nous ne pouvons conclure sur ce point. D'autre part, les ions cuivriques complexent la matière organique (LEWIS, 1961) et devraient conduire à moins d'interférences que dans l'extrait de $\mathrm{CaCl}_{2}$.

\section{CONCLUSIONS}

Cette étude comparative a permis de vérifier que les divers milieux n'ont pas influencé de manière mesurable l'extraction des nitrates. On peut donc considérer que le rendement de l'extraction ne biaise pas l'interprétation des différences de dosage observées dans les divers milieux.

\section{Devarda}

Cette méthode a donné les mêmes résultats, aux imprécisions près, dans les divers milieux d'extraction. Dans tous les cas, nous avons retrouvé les ajouts de nitrates. Il est important cependant de signaler que l'utilisation d'un dispositif automatique de titration représente une garantie utile de la valeur du dosage. Celui-ci permet en effet d'apprécier le point final du dégagement d'ammoniac et de s'assurer que ce dégagement s'est fait dans de bonnes conditions. S'il est trop lent, cela peut signifier que la quantité d'alliage réducteur est trop faible ou que les conditions de $\mathrm{pH}$ ne sont pas bonnes par exemple.

\section{Colorimétries automatiques}

Dans l'extrait $\mathrm{KCl} 2 \mathrm{~N}$, elles ont donné des résultats comparables au DEVARDA, sans interférences mesurables. Elles présentent l'avantage de permettre une cadence d'analyse plus grande que le DEvarDA. Dans les extraits $\mathrm{CaCl}_{2}$ et $\mathrm{CuSO}_{4}$, des interférences ont été décelées ; les ions cuivriques en particulier jouent un rôle dans la réduction des nitrates en nitrites et la variabilité de leur teneur dans les extraits ne permet pas d'établir de gammes convenables pour étalonner l'analyseur. Dans la plupart de nos échantillons, la quasi-totalité des ions cuivriques ayant été retenue par le sol, un étalonnage de l'analyseur sur une gamme faite dans l'eau s'est révélé convenable. Mais ce résultat n'est certainement pas extrapolable à tous les types de sol.

Néanmoins, les erreurs relatives observées pour la méthode au cadmium ont été faibles dans les extraits $\mathrm{CaCl}_{2}$ et $\mathrm{CuSO}_{4}$ ainsi que, pour la méthode à l'hydrazine, dans l'extrait $\mathrm{CaCl}_{2}$, et, dans bien des applications, on pourra considérer que les dosages obtenus sont convenables.

\section{Potentiométrie}

Elle a systématiquement donné des résultats beaucoup plus élevés (jusqu'à 50 p. 100) que le DEVARDA lorsque nous avons utilisé l'électrode ORION 93-07, alors qu'une électrode ORION 92-07 nous a donné pleine satisfaction. L'abaissement du $\mathrm{pH}$ de l'extrait à une valeur comprise entre 4 et 4,5 ne modifie pas les résultats donnés par cette dernière électrode mais a amélioré ceux de l'électrode 93-07. Dans l'extrait $\mathrm{CaCl}_{2}$, toute erreur a ainsi pu être supprimée. Il apparaît qu'entre modèles d'électrodes, des différences de spécificité importantes influent sur le résultat et done sur le protocole d'analyse. Nous signalerons par ailleurs qu'entre 2 modules d'électrodes de la même sćrie 93-07, l'un reçu en 1978, l'autre en 1980, la sensibilité à certaines interférences signalées par leurs manuels d'instruction respectifs a considérablement varié. Il convient donc de vérifier la validité d'un protocole donné pour le type d'électrode que l'on possède, alors que la quasitotalité des références bibliographiques porterit sur l'ancien modèle d'électrode ORION 92-07.

Contrairement à ØIEN \& SELMER-OlSEN (1969), nous n'avons pu obtenir de résultats satisfaisants dans l'extrait $\mathrm{CuSO}_{4}$. Il est vrai que nous n'avons travaillé ni avec la même électrode, ni avec les mêmes types de sol. Ces auteurs ont étudié des sols tourbeux ou sableux probablement pauvres en calcaire alors que notre échantillonnage comporte une majorité de sols calcaires dont la teneur se trouve corrélée aux erreurs constatées. L'abaissement du $\mathrm{pH}$ à 4,5 n'a que partiellement éliminé les interférences et l'erreur résiduelle n'a pu être expliquée.

Il est important enfin de signaler une différence de répétabilité importante entre les méthodes étudiées. Le coefficient de variation des dosages (calculé sur une centaine de déterminations répétées 3 fois) est de 1,6 p. 100 pour la potentiométrie, 2,5 p. 100 pour la méthode automatique à l'hydrazine et 4 p. 100 pour la méthode automatique au cadmium. Cette caractéristique avantage la potentiométric dans les cas où la répétabilité est un facteur déterminant (calculs de bilans par exemple).

Reçu le 30 mai 1981 Accepté le 30 novembre 1981.

\section{RÉFÉRENCES BIBLIOGRAPHIQUES}

Bremner J. M., 1965. In black C. A., editor, Methods of Soil Analysis, part 2, Agronomy 9". American Society of Agronomy. Wisconsin, p. 1179.

Goodman D., 1976. Automatic apparatus for the determination of pH and nitrate in soils. Analyst, 101, 943-948.

Henriksen A., Selmer-Olsen A. R., 1970. Automatic methods for determining nitrate and nitrite in water and soil extracts. Analyst, 95, 514-518.

Lewis D. G., 1961. Determination of inorganic nitrogen in soil. $J$. Sci. Fd. Agric., 12, 735-742.

Mazoyer R., 1972. L'ionométrie, ses possibilités d'application au dosage de l'azote nitrique et de l'azote ammoniacal en agronomie. Ann. agron., 23, 673-684.
Milham P. J., Awad A. S., Paull R. E., Bull J. M., 1970. Analysis of plants, soils and waters for nitrate by using an ion-sensitive electrode. Analyst, 95, 751-757.

Øien A., Selmer-Olsen A. R., 1969. Nitrate determination in soil extracts with the nitrate electrode. Analyst, 94, 888-894.

Snell F. D., Snell C. T., 1949. Colorimetric methods of Analysis. D. Van Nostrand Company Inc. New York, vol. II, 804-805.

Stainton M. P., 1974. Efficient reduction column for use in the automated determination of nitrate in water. Analyt. Chem., $\mathbf{4 6}$, p. 1616.

Terrey D. R., 1966. An automatic absorptiometric method for the determination of nitrate. Anal. Chim. Acta, 34, 41-45.

Anonyme, 1978. Nitrates, An environmental assessment. National Academy of Sciences, Washington D.C., 152-155. 